\title{
Nanoscale
}

PAPER
View Article Online

View Journal I View Issue
Check for updates

Cite this: Nanoscale, 2018, 10, 4105

\section{Viable stretchable plasmonics based on unidirectional nanoprisms $\dagger$}

\author{
Ji-Eun Lee, (D) ${ }^{a}$ Choojin Park, (D) ${ }^{b}$ Kyungwha Chung, (D) a Ju Won Lim, (D) ${ }^{a}$ \\ Filipe Marques Mota, (D) ${ }^{a}$ Unyong Jeong (D)*c and Dong Ha Kim (D)*a
}

\begin{abstract}
Well-defined ordered arrays of plasmonic nanostructures were fabricated on stretchable substrates and tunable plasmon-coupling-based sensing properties were comprehensively demonstrated upon extension and contraction. Regular nanoprism patterns consisting of $\mathrm{Ag}$, $\mathrm{Au}$ and $\mathrm{Ag} / \mathrm{Au}$ bilayers were constructed on the stretchable polydimethylsiloxane substrate. The nanoprisms had the same orientation over the entire substrate $\left(3 \times 3 \mathrm{~cm}^{2}\right)$ via metal deposition on a single-crystal microparticle monolayer assembly. The plasmonic sensor based on the Ag/Au bilayer showed a 6 -fold enhanced surface enhanced Raman scattering signal under $20 \%$ uniaxial extension, whereas a 3 -fold increase was observed upon $6 \%$ contraction, compared with the Au nanoprism arrays. The sensory behaviors were corroborated by finitedifference time-domain simulation, demonstrating the tunable electromagnetic field enhancement effect via the localized surface plasmon resonance coupling. The advanced flexible plasmonic-coupling-based devices with tunable and quantifiable performance herein suggested are expected to unlock promising potential in practical bio-sensing, biotechnological applications and optical devices.
\end{abstract}

Received 8th November 2017 Accepted 18th January 2018

DOI: $10.1039 / c 7 n r 08299 a$

rsc.li/nanoscale
Kim et al. fabricated meta-materials consisting of period- and symmetry-tunable self-assembled nanopatterns using blockcopolymer lithography onto poly(dimethyl siloxane) (PDMS) films. The precise inter-distance manipulation of the patterns by inhomogeneous thermal shrinkage was monitored following the refractive index of the resulting metamaterials, essential for an effective enhancement of refractive index. ${ }^{21}$ Aksu et al. reported the development of high-quality bow tie antennas based on high-resolution nanostencil lithography on a PDMS substrate. The optical responses of the antenna arrays were shown to be tuned by the mechanical stretching of the stretchable substrate. The meta-structures were shown to be highly durable, with the stretchable nature of the selected substrate desirable for active tuning of plasmonic resonances. ${ }^{17}$ Details of the growing process of gold nanoparticles (AuNPs) onto the PDMS template were later provided by Cataldi et al. ${ }^{19}$ The synergy between the controlled growth of AuNPs and the mechanical strain was claimed to lead to a drastic shift of the plasmon band accompanied by a color change of the sample.

However, so far the plasmonic nanostructures on stretchable substrates have been fabricated by complicated procedures and the directional arrangement of the structures was ordered in a short distance, being random over a large area. ${ }^{18-20}$ It is noted that any properties obtained from randomly arranged plasmonic nanostructures may lack reproducibility because the apparent strain applied on the substrate should cause different local geometrical changes according to the local orientations of the nanostructures. This non-uniform 
strain would intervene in the correct understanding of the effect of geometrical change of the nanostructures on the plasmonic properties. Thus, it is a valuable task to develop viable stretchable plasmonic systems in which the plasmonic-coupling properties are tailored identically in the entire range of the substrate. Herein, we demonstrated tunable surface enhanced Raman scattering (SERS) activities based on plasmonic-coupling in well-defined and highly oriented periodic metal nanoprisms on stretchable substrates. SERS signals were then systematically investigated using composition-controlled metal nanoprism arrays under uniaxial elongation and compression.

\section{Experimental section}

\section{Materials}

Anhydrous ethanol was purchased from DAE JUNG chemicals and styrene, polyvinylpyrrolidone (PVP, $M_{\mathrm{w}} 55$ 000), and azobisisobutyronitrile (AIBN) were purchased from Sigma-Aldrich. Polydimethylsiloxane kit (Pre-polymer and crosslinker, Sylgard 184) was purchased from Dow Corning.

\section{Preparation of polystyrene particles}

Polystyrene (PS) beads were synthesized by controlled dispersion polymerization. After purging the stabilized anhydrous ethanol and PVP (0.98 g, $1.2 \mathrm{mM}$ ) under an Ar gas flow for $30 \mathrm{~min}$ at $70{ }^{\circ} \mathrm{C}$, AIBN (0.0312 g) initiator was added, followed by quick injection of purified styrene monomers (3.4 mL, $1 \mathrm{M})$ which had been filtered with alumina. After polymerization with homogeneous stirring at $70{ }^{\circ} \mathrm{C}$ for $20 \mathrm{~h}$, PS beads with an average diameter of about $1 \mu \mathrm{m}$ were obtained. The particles were centrifuged and washed three times and then dried in a vacuum chamber at room temperature for $12 \mathrm{~h}$.

\section{Preparation of PS monolayer on PDMS substrate}

A thin and thick PDMS substrate $(\sim 0.3 \mathrm{~mm}$ thickness and $\sim 6 \mathrm{~mm}$ thickness) was prepared by mixing a prepolymer with a curing agent (Sylgard 184, Dow Corning) in a $10: 1$ weight ratio at room temperature. The Si-wafer was coated with PDMS by spin-coating at $500 \mathrm{rpm}$ for $10 \mathrm{~s}$ and then cured at $70^{\circ} \mathrm{C}$ for $5 \mathrm{~h}$. The rubber-coated Si substrate was fixed on a stage. After placing PS particle powder, the bottom PDMS substrate was covered by the rubbing process. A PS monolayer array of diameter $1 \mu \mathrm{m}$ was formed on the PDMS substrate.

\section{Preparation of metal nanoprism arrays}

The metal nanostructures were prepared by depositing a $100 \mathrm{~nm}$-thick metal film on the PS monolayer array by thermal evaporation. Metal nanofilms were deposited using a thermal evaporation system (GVEV7200-1206) from GV-Tech Corporation at $0.1-0.2 \AA \mathrm{s}^{-1}$. Thus, a monolayer of the metalPS array was formed on the elastic PDMS substrate. The metal film and PS monolayer were detached with scotch tape from the metal-PS array.

\section{FDTD simulation}

Finite-difference time-domain (FDTD) simulation by the Lumerical software was used for the calculation of the nearfield electromagnetic field distributions of metal nanostructures arrays with a plane wave source at $633 \mathrm{~nm}$. The simulation domain for the $z$ direction was used by a perfectly matched layer (PML) to absorb all the field propagating to the outer, while the $x$ and $y$ directions were periodic boundary conditions to replicate the metal nanostructure array. The refractive indexes of $\mathrm{Au}$ and $\mathrm{Ag}$ were used from the data of Palik. The size of mesh was $2 \mathrm{~nm}$ for all directions with a plane wave input source of 200 to $1200 \mathrm{~nm}$.

\section{Instruments and characterization}

Transmission electron microscopy (TEM) measurements were carried out using a JEOL JSM2100-F microscope operated at 10 $\mathrm{kV}$. UV-vis absorption spectra were recorded on a Varian Technologies Cary 5000 with diffuse reflectance accessories (DRA). The metal nanostructures were observed using a JEOL JSM6700-F scanning electron microscope (SEM). Atomic force microscopy (AFM) studies on the surface morphologies of the metal nanostructure on the PDMS substrate were performed with a Digital Instruments Dimension 3100 scanning force microscope in tapping mode. Raman spectra were obtained with a Raman Spectrometer from HORIBA Jobin Yvon at an excitation wavelength of $633 \mathrm{~nm}$ and a Nikon microscope with a $\times 50$ objective lens and a numerical aperture (NA) of 0.75 . The acquisition and accumulation time of each spectrum were $10 \mathrm{~s}$ and $5 \mathrm{~s}$, respectively. The scan range was 200 to $2000 \mathrm{~cm}^{-1}$. SERS substrates were cut into $3 \times 3 \mathrm{~cm}^{2}$ pieces and incubated in $1 \mathrm{mM} p$-aminothiolpheol ( $p$-ATP) solutions and washed with ethanol, prior to measurements. $p$-ATP was used as the SERS probe with two critical Raman peaks at 1076 and $1140 \mathrm{~cm}^{-1}$.

\section{Results and discussion}

The overall procedure to demonstrate viable flexible plasmonics is schematically illustrated in Fig. 1a. Regular metal nanopatterns were constructed via colloidal lithography on a unidirectional monolayer assembly of microparticles following the procedure recently reported in our previous study. ${ }^{24}$ The assembled monolayer allowed for simple and reproducible deposition of metallic nanoprism arrays with the same direction over the entire substrate. Firstly, dried PS bead powders were sandwiched in between two PDMS substrates and uniaxially rubbed with slight palm pressure, leading to hexagonallypacked arrays of PS beads (Fig. S1 $\dagger$ ). ${ }^{24}$ Three different types of metal nanoprism arrays composed of a $\mathrm{Ag}, \mathrm{Au}$ and $\mathrm{Ag} / \mathrm{Au}$ double layer were prepared by thermal vacuum evaporation onto polystyrene (PS) bead monolayer arrays as model plasmonic nanostructures. Then, the PS monolayer was detached from the metal-PS array using scotch tape, leaving the metal nanoprism arrays remaining on the PDMS substrate. The Ag, $\mathrm{Au}$ and $\mathrm{Ag} / \mathrm{Au}$ bilayer nanoprism arrays were subjected to two strain components in extension and contraction states. The 

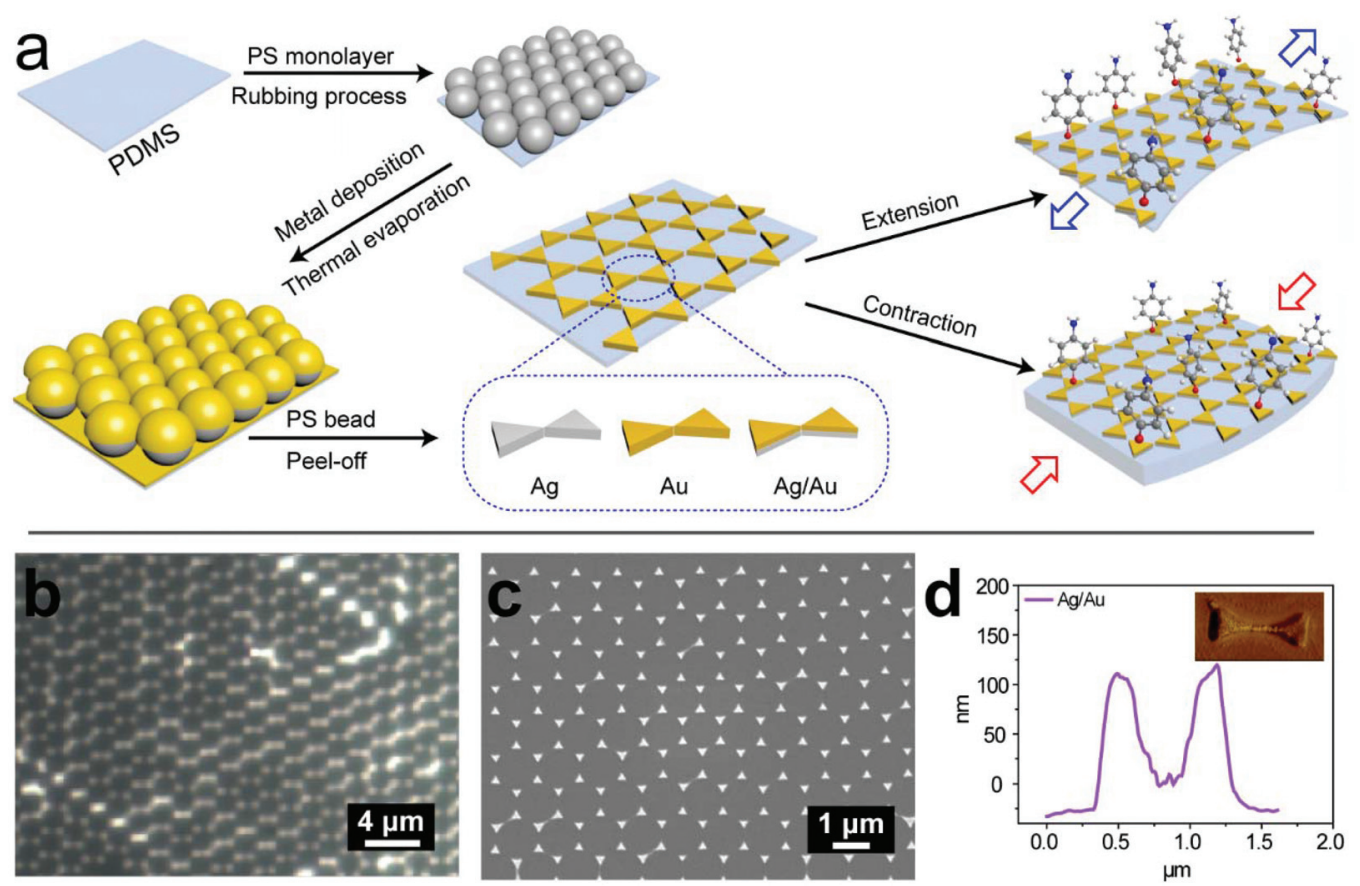

Fig. 1 Schematic diagram for the stepwise fabrication procedure of metal nanostructure arrays on stretchable PDMS substrates and for extension/ contraction on the thin and thick PDMS films (a). Optical microscopy image (b), SEM image (c) and cross-sectional AFM profile (d) for the morphology of Ag/Au bilayers on stretchable PDMS substrates. The inset in (d) is a height contrast AFM image.

extension was performed with thinner PDMS substrates, while the contraction was carried out with thicker PDMS substrates to ensure the dimensional stability against rupture. $p$-ATP was employed as the SERS reporter on metal nanoprism surfaces.

The surface morphology of the $\mathrm{Ag} / \mathrm{Au}$ bilayer nanoprisms was characterized using SEM and AFM images as shown in Fig. 1b-d. Regular and uniform metal nanopatterns were obtained. Ag and Au were sequentially deposited on the microparticle monolayer assembly. The thickness of both $\mathrm{Ag}$ and $\mathrm{Au}$ layers was $50 \mathrm{~nm}$ and the edge length was $250 \mathrm{~nm}$. The change in the morphology of metal nanopatterns was monitored upon contraction and extension. The gap-distances between the metal nanoprisms inthe extended and contracted states were measured by optical microscopy imaging. The distances were $-100,0,209$ and $555 \mathrm{~nm}$ at strains of $-6,0,20$ and 100\%, respectively (see the ESI in Fig. S2 $\dagger$ ). Dimensional characterization of the nanoprisms of $\mathrm{Ag}$ and $\mathrm{Au}$ are provided in the ESI (Fig. S3†). The thickness of the metal nanoprisms was measured to be $\sim 100 \mathrm{~nm}$, according to the cross-sectional profiles obtained from the corresponding AFM images of selected areas.

The optical properties of a defined unit of the neighboring nanostructures within a $4.9 \mu \mathrm{m}^{2}$ surface area of the substrate were assessed through the corresponding transmittance spectra (Fig. S4†). The obtained spectra conspicuously yielded strong absorption peaks within the $500-700 \mathrm{~nm}$ wavelength range. The optical properties of the as-prepared metal nanoprism arrays and those at different extension ratios were studied for $\mathrm{Ag}$, $\mathrm{Au}$ and $\mathrm{Ag} / \mathrm{Au}$ as shown in the UV-Vis absorp- tion spectra (Fig. 2). Two main LSPR bands were observed at $\lambda=\sim 413$ and $\sim 910 \mathrm{~nm}$ in the absorption spectrum of Ag nanoprism arrays in which the latter is ascribed to the coupling band. Interestingly, the plasmon-coupling-induced band at $\sim 910 \mathrm{~nm}$ showed a red-shift behaviour $(\sim 1011 \mathrm{~nm})$ at the extension ratio of $20 \%$, and then it was blue-shifted to $\sim 971 \mathrm{~nm}$ concurrent with a decrease in intensity at the extension ratio of $100 \%$ (Fig. 2a). A similar trend was also observed for the $\mathrm{Au}$ and $\mathrm{Ag} / \mathrm{Au}$ prism arrays. The Au nanoprism arrays showed characteristic absorption bands at $\lambda=\sim 629$ and $\sim 910 \mathrm{~nm}$. At the extension ratio of $20 \%$, the plasmon band at $629 \mathrm{~nm}$ remained constant while a red-shift from $\sim 910$ to $\sim 983 \mathrm{~nm}$ was observed (Fig. 2b) for the coupling band. For further stretching at $100 \%$ extension ratio, this band was blueshifted at $\sim 917 \mathrm{~nm}$ with a decreased intensity.

In the case of the $\mathrm{Ag} / \mathrm{Au}$ bilayer nanoprism arrays, three absorbance bands were observed at $\lambda=\sim 419, \sim 540$ and $\sim 937 \mathrm{~nm}$. The prepared $\mathrm{Ag} / \mathrm{Au}$ bilayer nanoprism arrays are expected to exhibit at least four absorbance peaks such as two characteristic peaks of Ag and $\mathrm{Au}$ and two coupling bands. It is assumed that the Au coupling band was observed as a shoulder peak, hidden behind the Ag coupling band. Upon extension of the $\mathrm{Ag} / \mathrm{Au}$ bilayer nanoprism arrays by $\sim 20 \%$, the band position changed from $\sim 937$ to $\sim 1001 \mathrm{~nm}$. After further stretching, the absorption band was blue-shifted to $\sim 913 \mathrm{~nm}$. Absorbance bands at $\lambda=\sim 418, \sim 533, \sim 913$ and $\sim 1001 \mathrm{~nm}$ were ascribed to the plasmon properties of $\mathrm{Ag}$ and $\mathrm{Au}$ and the corresponding $\mathrm{Ag}$ and $\mathrm{Au}$ of the neighbouring nanostructures at an extension ratio of $100 \%$, respectively (Fig. 2 c). 


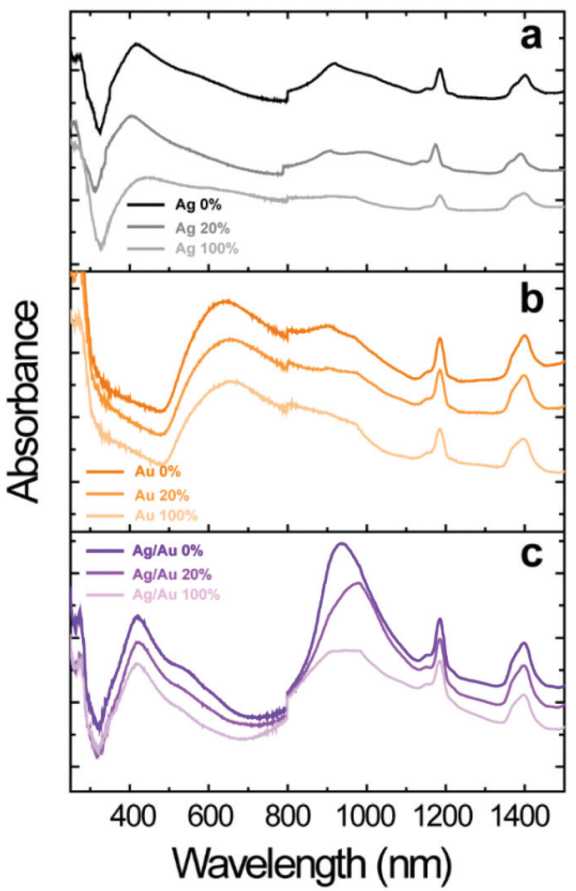

Fig. 2 UV-Vis spectra of metal nanoprism arrays observed at different extension ratios obtained from (a) Ag nanoprism arrays, (b) Au nanoprism arrays, and (c) $\mathrm{Ag} / \mathrm{Au}$ bilayer nanoprism arrays.

Both $\mathrm{Ag}$ and $\mathrm{Au}$ nanoprism arrays exhibit two LSPR peaks in the corresponding obtained spectra. The assignment of the observed LSPR bands of both Ag and Au nanoprism arrays to the quadrupolar and dipolar oscillations of the free electrons followed previous literature reports. ${ }^{25}$ As the dipole resonance is found to be very intense, and its peak wavelength is extremely sensitive to the height, edge length, and tip sharpness of the nanoprism, and the distance between structures, change in the morphology of the rationally designed nanoprisms was previously observed to lead to a change in the LSPR band ascribed to the dipolar plasmon mode. In contrast, the intensity of the quadrupole resonance was found to be much weaker relative to the dipole resonance in the single particles spectra than in the ensemble averaged spectrum, in accordance with our observations. While presumed to overlap with the dipolar oscillation band, the Bragg diffraction mode expected to be observed at $910 \mathrm{~nm}$ emerges from the hierarchical arrangement of nanoprism arrays. Upon mechanical manipulation within the evaluated extension range, the architectural order was preserved.

However, as neighbouring nanoprisms are distantiated, a shift in the Bragg diffraction mode is to be expected. With a continuous strain increase, the dipolar resonance was continuously skewed toward higher wavelength ranges. ${ }^{26,27}$ Conversely, the quadrupolar resonance showed a relatively similar band position within the evaluated range in accordance with the fact that the latter has been shown to respond to local refractive index changes. ${ }^{28,29}$ At the highest strain levels, both dipolar resonance and diffraction peaks are observed at longer wavelengths up to $c a .1100 \mathrm{~nm}$.
In the case of $\mathrm{Ag}$, the two main LSPR bands observed at $\lambda=\sim 413$ and $\sim 910 \mathrm{~nm}$ were ascribed to the occurrence of quadrupolar and dipolar oscillations of the free electrons at the nanoprisms with diffraction mode, respectively. Similarly, both bands were observed at $\lambda=\sim 629$ and $\sim 910 \mathrm{~nm}$ for the $\mathrm{Au}$ nanopattern. For the $\mathrm{Ag} / \mathrm{Au}$ bilayer arrays, all LSPR bands were observed at the four positions previously mentioned. The modes were confirmed to be dependent on the individual distance between metal nanoprisms. The red-shift and subsequent blue-shift behaviours upon $\sim 20 \%$ and $\sim 100 \%$ extensions, respectively, observed from all the three different types of metal nanopatterns, can be interpreted as follows. Upon extension of the periodic prism arrays, an increase of the average distance between neighbouring prisms was in fact accompanied by an eventual decrease of the distance along the perpendicular direction to which the stress was applied. Most importantly, upon a $\sim 20 \%$ extension for example, only a minor change less than $1 \%$ in the perpendicular direction could be found as is evidenced in the representative optical images of these metal nanoprisms (Fig. S2†). Only upon a $\sim 100 \%$ extension could the variation in the distance of the nanoprisms be noticeable. Such a complex deformation behaviour is assumed to affect the plasmonic coupling phenomenon. Based on the observed experimental results, it is assumed that the latter effect was dominant at $20 \%$ extension. On the contrary, the absolute distance between neighbouring prisms becomes sufficiently large, leading to the blue-shift of the overall LSPR properties. In order to investigate the correlation between the structural alteration and LSPR behaviour more rigorously, reflectance spectra of Ag, Au and $\mathrm{Ag} / \mathrm{Au}$ bilayer nanoprism arrays at different extension ratios were also studied (Fig. S5 $\dagger$ ). The reflectance spectra of metal nanoprisms at contraction ratios of $0 \%$ and $-6 \%$ were obtained (Fig. S6 $\dagger$ ). The $\mathrm{Ag} / \mathrm{Au}$ bilayer spectrum unveiled the combined features of the two metal components, with an overall lower peak intensity compared with $\mathrm{Ag}$ and $\mathrm{Au}$ nanoprism arrays in the evaluated wavelength range. The $\mathrm{Ag} /$ $\mathrm{Au}$ bilayer nanoprism arrays were therefore concluded to possess superior transmission and absorption properties compared to individually evaluated $\mathrm{Ag}$ and $\mathrm{Au}$ nanoprism arrays. ${ }^{25}$ In addition, the orientations of the metal nanoprism arrays are equivalent in both extension and contraction along the two directions, according to the designed geometrical configuration of arrays of paired prisms. Along the polarization dependent directions during extension and contraction, the resulting optical properties are the averaged result at a large scale of extension/contraction in two mutually orthogonal directions. In this context, the orientations of the metal nanoprism arrays are equivalent in both extension and contraction along the two directions. Also, the polarization dependent resonances represent the same properties at various aspects of any strength positions.

We have then investigated the SERS spectra obtained from three types of metal nanoprism arrays on thin and thick PDMS substrates upon excitation and contraction with a $633 \mathrm{~nm}$ laser. A reference SERS spectrum of $p$-ATP molecules on a 

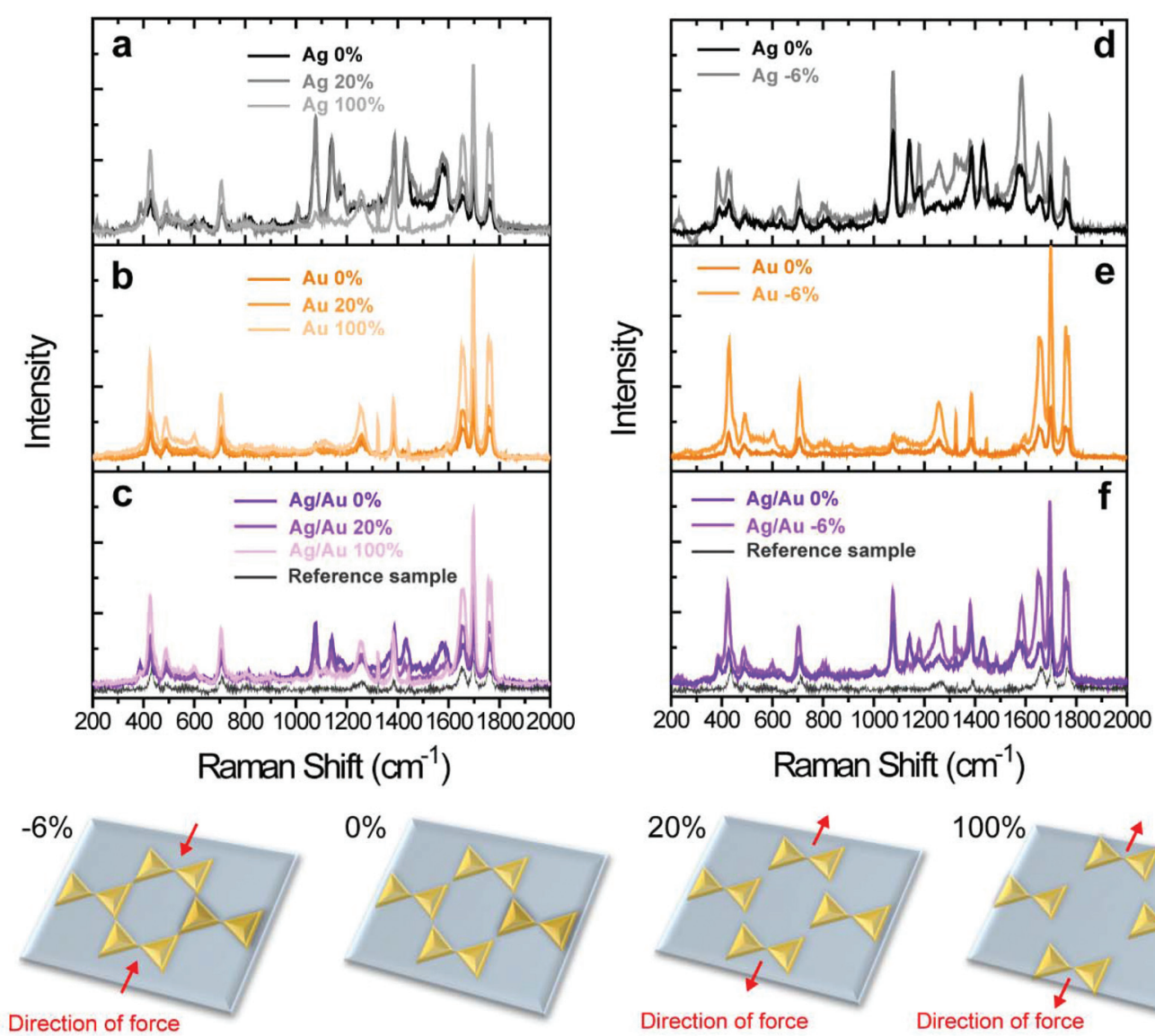

Direction of force

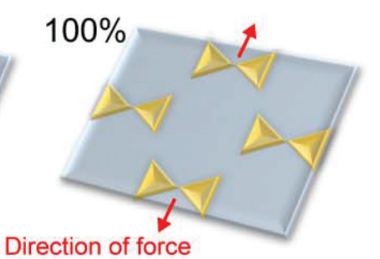

Fig. 3 SERS spectra with extension state and contraction state of $-6,0,20$ and 100\%. (a) and (d) Ag, (b) and (e) Au, (c) and (f) Ag/Au bilayer nanoprism arrays on the PDMS substrate. Schematic of the change in the morphology of metal nanopatterns upon contraction and extension in the direction of force. Reference sample is compared in (c) and ( $f$ ), the SERS spectrum of the adsorbed $p$-ATP molecules on the PDMS without metal nanoprism arrays.

PDMS substrate in the absence of metal nanoprism arrays is depicted in Fig. S7.† The five bands at 431, 708, 1 655, 1699 and $1760 \mathrm{~cm}^{-1}$ were attributed to the stretching and vibration modes of PDMS, whereas no Raman signals were observed from the $p$-ATP molecules. Fig. 3 shows the SERS spectra of $p$-ATP molecules assembled on the metal nanoprism arrays by covalent binding upon extension and contraction, respectively. All the spectra exhibit five peaks at $1076 \mathrm{~cm}^{-1}, 1140 \mathrm{~cm}^{-1}$, $1181 \mathrm{~cm}^{-1}, 1383 \mathrm{~cm}^{-1}$ and $1436 \mathrm{~cm}^{-1}$, corresponding to the vibrational mode of $\mathrm{C}-\mathrm{S}, \mathrm{C}-\mathrm{H}$ stretching of $\mathrm{b}_{2}$ mode, $\mathrm{C}-\mathrm{H}$ bending mode of $\mathrm{a}_{1}$ mode, $\mathrm{C}-\mathrm{C}$ bending of $\mathrm{b}_{2}$ mode and $\mathrm{C}-\mathrm{C}$ stretching of $b_{2}$ mode, respectively. In addition, the Raman signals of two $b_{2}$ modes at 1383 and $1436 \mathrm{~cm}^{-1}$ were observed, corresponding to $\mathrm{N}=\mathrm{N}$ stretching, which can be ascribed to the oxidative transformation of $p$-ATP to DMAB. ${ }^{19,30}$ Consequently, strong peak intensities at $1076 \mathrm{~cm}^{-1}$ and $1140 \mathrm{~cm}^{-1}$ were selected to observe the enhancement of Raman signal.

The SERS intensity of $p$-ATP onto metal prisms at $1076 \mathrm{~cm}^{-1}$ obtained from $\mathrm{Ag}, \mathrm{Au}$ and $\mathrm{Ag} / \mathrm{Au}$ bilayer nanoprisms was 60000,10000 and 45000 counts, respectively. Using the same conditions, the SERS signal at $1140 \mathrm{~cm}^{-1}$ from Ag,
$\mathrm{Au}$ and $\mathrm{Ag} / \mathrm{Au}$ bilayer nanoprisms was 58000,7000 and 50000 counts, respectively. At the extension ratio of $20 \%$, the Raman signal of Ag nanoprisms strongly increased with the increasing gap-distance between the Ag nanoprisms, the mechanism of which needs to be clarified further. It was observed that the overall SERS signal from Ag nanoprisms was stronger than that from $\mathrm{Au}$ because $\mathrm{Ag}$ has a larger scattering cross section than $\mathrm{Au} .{ }^{31} \mathrm{Au}$ and $\mathrm{Ag} / \mathrm{Au}$ bilayer nanoprisms showed a slight decrease in SERS intensity distributions at the extension state of $20 \%$. At the extension of $100 \%$, the SERS intensity of $\mathrm{Ag}, \mathrm{Au}$ and $\mathrm{Ag} / \mathrm{Au}$ bilayer nanoprisms at $1076 \mathrm{~cm}^{-1}$ and $1140 \mathrm{~cm}^{-1}$ was substantially decreased (Fig. 3a-c). Fig. 3d-f show the Raman signal of metal nanoprism arrays at 0 and $-6 \%$ contraction states. The intensity of the SERS signal at $1076 \mathrm{~cm}^{-1}$ obtained from all metal nanoprism arrays at $-6 \%$ contraction is greater than that at $0 \%$ strain due to the decreased gap-distance between the metal nanoprisms. The SERS peak at $1140 \mathrm{~cm}^{-1}$ in the contraction of $-6 \%$ was observed to show a decrease in SERS intensity compared to that at $0 \%$ strain in both $\mathrm{Ag}$ and $\mathrm{Ag} / \mathrm{Au}$ nanoprism arrays including the $\mathrm{Ag}$ material. In contrast, the SERS peaks at 1176,1383 , and $1436 \mathrm{~cm}^{-1}$ were higher compared to the initial state. 
The measured reflectance spectra of all samples under stress-free conditions and under extension and contraction mechanical manipulation yielded characteristic absorption bands in the $600-700 \mathrm{~nm}$ region. In agreement with previous reports on two-dimensional substrates the found match between the laser excitation wavelength $\lambda_{\mathrm{ex}}$ and the absorption range of each sample was considered suitable to dictate the SERS enhancement herein evaluated. ${ }^{32}$ The enhancement of the $b_{2}$ mode with visible excitation was interpreted in terms of a metal-to-molecule charge-transfer mechanism, which largely depends on the energy of excitation lights. In addition, the non-reactivity of $p$-ATP under a $\mathrm{N}_{2}$ atmosphere suggests that the appearance of the Raman bands at 1383 and $1436 \mathrm{~cm}^{-1}$ should be due to the conversion of $p$-ATP to DMAB. ${ }^{30}$ Dimerization of $p$-ATP into DMAB is induced by efficient energy transfer from surface plasmon resonance to the surface adsorbed $p$-ATP. From these results two main conclusions could be drawn. Firstly, the intensity of the corresponding SERS signal of the $\mathrm{C}-\mathrm{H}$ bending and $\mathrm{C}-\mathrm{H}$ stretching was assumed to be a function of the orientation of the molecules on the SERS substrate.

In addition, our results have shed light on the electromagnetic effect and charge transfer mechanism between the molecules and metal. The enhancement of $b_{2}$ modes of the obtained Raman spectra was interpreted as a result of a metalto-molecule charge transfer mechanism, largely depending on the energy of the selected excitation light. ${ }^{33-35}$

The above-described SERS results are summarized in Fig. 4. In brief, the $\mathrm{Ag}$ nanoprisms showed higher Raman intensity than $\mathrm{Au}$ and $\mathrm{Ag} / \mathrm{Au}$ nanoprisms, while the result obtained from the $\mathrm{Au}$ nanoprism was more reproducible than $\mathrm{Ag}$ and $\mathrm{Ag} / \mathrm{Au}$ bilayer nanoprisms at various strain ratios. $\mathrm{Ag} / \mathrm{Au}$ nanoprism arrays showed greater SERS activity than Au nanoprism arrays and better reproducibility than $\mathrm{Ag}$ nanoprism arrays. These results reflect the inherent better stability
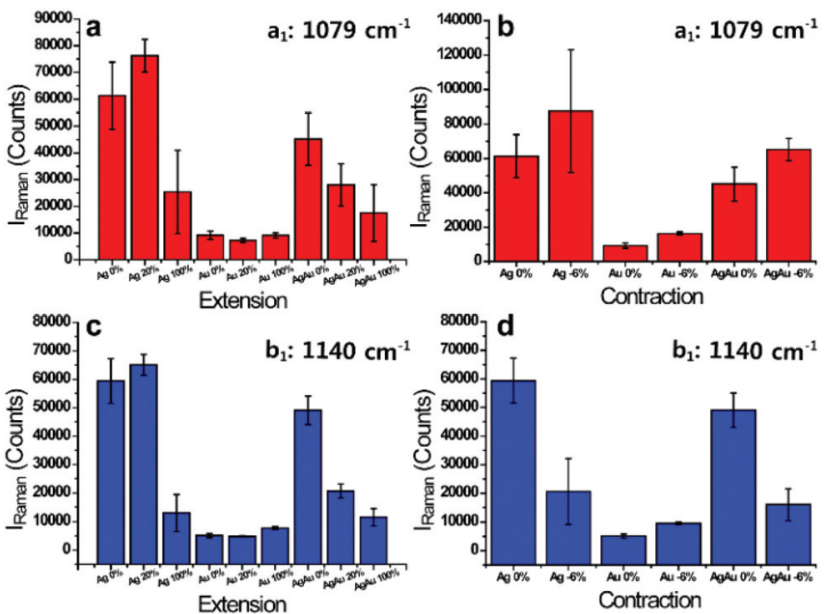

Fig. 4 SERS intensity of two critical Raman peaks at 1076 and $1140 \mathrm{~cm}^{-1}$ with extension $(a, c)$ and contraction $(b, d)$ states. Error bars are standard deviations from independently measured results. of $\mathrm{Au}$ and higher extinction coefficient of Ag. Conclusively, bimetallic $\mathrm{Ag} / \mathrm{Au}$ nanoprisms may retain the combined synergy of higher sensitivity of $\mathrm{Ag}$ and enhanced stability of $\mathrm{Au}$, i.e., consistently exhibiting higher SERS enhancement factor and better reproducibility than monometallic nanoprisms.

To support the experimental observations, the distribution of hot-spots was investigated by finite-difference time-domain (FDTD) simulation. Fig. 5 shows the electromagnetic near-field distributions along the $x$ and $y$ directions with different nanoprism arrays and strain ratios simulated using a plane wave source at $633 \mathrm{~nm}$ wavelength. Possible irregularities or the presence of defects in our array were considered minor at a large scale. Similarly, the use of PS beads with the same size leading to different nanoprism arrays with a varying gap-distance between the metal nanoprisms was considered uniform. The assumptions have been reflected in the representative SEM images, AFM images, and optical microscopy images illustrating the quality of the arrays herein prepared. A varying gap-distance between the metal nanoprisms resulted in different electromagnetic fields in each case. In particular, all metal nanoprism arrays under stress-free conditions yielded bands at 400-600 $\mathrm{nm}$ and 800-1000 nm wavelength (Fig. S8†). As shown in the results, the most expected divergence between the simulated and the prepared samples is the presence of the PDMS substrate in the latter case. The optical properties of the latter are not taken into account during the simulation, which is expected to have an effect on the entire range herein analyzed. In addition, the assembling of adjacent hetero-nanostructures with different characteristics, such as angular momentum or line shape, is believed to result in strong interactions additionally shown to be a function of inter-particle distance. The interactions have been demonstrated to result in energy shifts and avoided crossings of the plasmon modes as the constituent nanoparticles coalesce into the dimer struc-

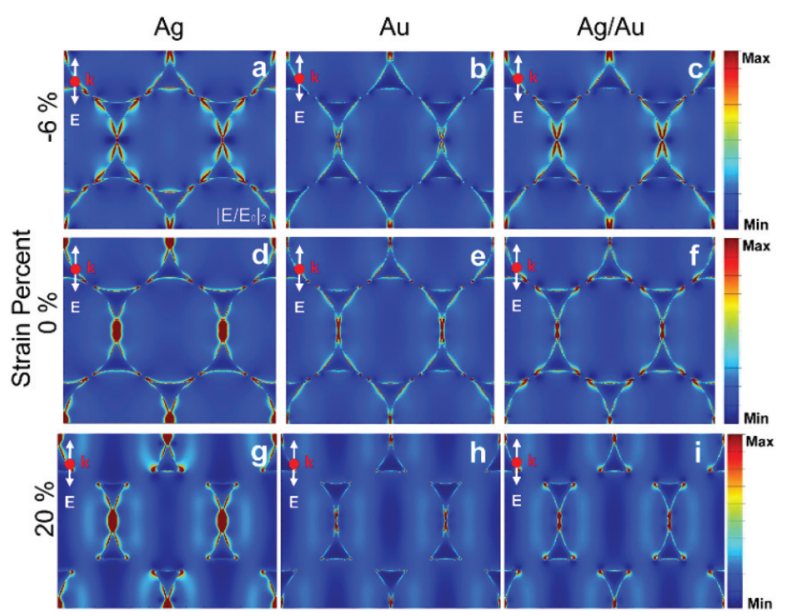

Fig. 5 The FDTD results of near field electromagnetic field distributions with different metal and strain components, respectively. Ag nanoprism arrays (a) $-6 \%$, (d) $0 \%$ and (g) $20 \%$; Au nanoprism arrays (b) $-6 \%$, (e) $0 \%$ and (h) $20 \%$; Ag/Au bilayer nanoprism arrays (c) $-6 \%$, (f) $0 \%$ and (i) $20 \%$ (plane wave source at $633 \mathrm{~nm}$ wavelength). 
ture. ${ }^{36,37}$ The simulated electromagnetic field distribution also depends on the strain ratio and the type of metal. The maximum $\left|E / E_{0}\right|^{2}$ value for the three $\mathrm{Ag}$, $\mathrm{Au}$ and $\mathrm{Ag} / \mathrm{Au}$ nanoprisms was measured to be 1620,278 and 545, respectively, at the extension ratio of $0 \%$.

Upon extension by $20 \%$, the maximum $\left|E / E_{0}\right|^{2}$ field distributions of Ag, $\mathrm{Au}$ and $\mathrm{Ag} / \mathrm{Au}$ bilayer nanoprisms were 2 970, 199 and 612, respectively, while at the contraction ratio of $-6 \%$, the value was 162, 282 and 443, respectively. These results are in accordance with the enhanced SERS activity for each material.

In the contraction state of $\mathrm{Ag}$ nanoprism arrays, similarly prominent hot-spots were observed at all vertexes of the nanostructure, in the electromagnetic field distribution. The edge area adjacent to the vertexes in this case was further confirmed to show a stronger electromagnetic field than under other strain conditions. Similarly, in the case of Au nanoprism arrays, the hot-spot appeared at all vertexes at the 20 and $0 \%$ extensions. However, Au exhibited remarkably lower electromagnetic field density than the Ag nanoprism arrays, corroborating the abovementioned SERS results. For the Ag/Au bilayer nanoprism arrays intense hot-spots were produced by near field coupling. In the case of $0 \%$ strain and $20 \%$ extension ratio, a prominent hot-spot appeared at all vertexes in the electromagnetic field distribution. At the contraction ratio of $-6 \%$ and as observed with $\mathrm{Ag}$, the intense hot-spots were further observed in the edge areas adjacent to the vertexes of the nanostructure. Detailed analysis on the field distributions reveals three characteristics: (i) the electromagnetic enhancement is highly localized at the vertices of the triangles, which is evident from the field distribution of all the structures. (ii) The highest electromagnetic enhancement is localized at the vertexes corresponding to the center of the ribbon nanostructures. (iii) The electromagnetic field is the strongest for the $\mathrm{Ag}$ nanoprism arrays at $20 \%$ extension, and is stronger than that at $0 \%$ strain.

\section{Conclusions}

We have demonstrated tunable near field electromagnetic field enhancement and viable plasmonic-coupling-based sensing using highly oriented periodic $\mathrm{Ag}$, $\mathrm{Au}$ and $\mathrm{Ag} / \mathrm{Au}$ nanoprism arrays with two-strain components on the PDMS substrate. It was demonstrated that the extension and contraction of the stretchable PDMS substrate can tune the plasmonic properties to maximize SERS signals. We found that an optimized configuration obtained from $\mathrm{Ag} / \mathrm{Au}$ nanoprism arrays with a specific distance and contraction ratio of $-6 \%$ provided enhanced SERS activity by 3-fold compared with the Au nanoprism arrays. It was also confirmed that $\mathrm{Ag} / \mathrm{Au}$ nanoprism arrays showed better reproducibility than $\mathrm{Ag}$ nanoprism arrays. Thus, the stimuli-responsive stretchable nanostructures with SPR coupling-based tunability and function established in this study can be explored for diverse advanced plasmonic-coupling-based applications.

\section{Conflicts of interest}

There are no conflicts to declare.

\section{Acknowledgements}

This study was supported by the National Research Foundation of Korea Grant funded by the Korean Government (2017R1A2A1A05022387). U. Jeong acknowledges the support from Samsung Research Funding Center of Samsung Electronics under Project Number SRFC-MA1301-07.

\section{References}

1 E. Hutter and J. H. Fendler, Exploitation of localized surface plasmon resonance, Adv. Mater., 2004, 16, 16851706.

2 S. A. Maier and H. A. Atwater, Plasmonics: Localization and guiding of electromagnetic energy in metal/dielectric structures, J. Appl. Phys., 2005, 98, 011101.

3 E. Petryayeva and U. J. Krull, Localized surface plasmon resonance: nanostructures, bioassays and biosensing-a review, Anal. Chim. Acta, 2011, 706, 8-24.

4 K. A. Willets and R. P. Van Duyne, Localized surface plasmon resonance spectroscopy and sensing, Annu. Rev. Phys. Chem., 2007, 58, 267-297.

5 K. M. Mayer and J. H. Hafner, Localized Surface Plasmon Resonance Sensors, Chem. Rev., 2011, 111, 3828-3857.

6 J. E. Lee, K. Chung, J. Lee, K. Shin and D. H. Kim, In Situ Studies of Surface-Plasmon-Resonance-Coupling Sensor Mediated by Stimuli-Sensitive Polymer Linker, Adv. Funct. Mater., 2015, 25, 6716-6724.

7 J.-E. Lee, K. Chung, Y. H. Jang, Y. J. Jang, S. T. Kochuveedu, D. Li and D. H. Kim, Bimetallic multifunctional core@shell plasmonic nanoparticles for localized surface plasmon resonance based sensing and electrocatalysis, Anal. Chem., 2012, 84, 6494-6500.

8 D. X. Li, J. F. Zhang, Y. H. Jang, Y. J. Jang, D. H. Kim and J. S. Kim, Plasmonic-Coupling-Based Sensing by the Assembly and Disassembly of Dipycolylamine-Tagged Gold Nanoparticles Induced by Complexing with Cations and Anions, Small, 2012, 8, 1442-1448.

9 Y. H. Jang, K. Chung, L. N. Quan, B. Spackova, H. Sipova, S. Moon, W. J. Cho, H.-Y. Shin, Y. J. Jang, J.-E. Lee, S. T. Kochuveedu, M. J. Yoon, J. Kim, S. Yoon, J. K. Kim, D. Kim, J. Homola and D. H. Kim, Configuration-controlled Au nanocluster arrays on inverse micelle nano-patterns: versatile platforms for SERS and SPR sensors, Nanoscale, 2013, 5, 12261-12271.

10 Y. Chen, D. Yang, Y. J. Yoon, X. Pang, Z. Wang, J. Jung, Y. He, Y. W. Harn, M. He, S. Zhang, G. Zhang and Z. Lin, Hairy Uniform Permanently Ligated Hollow Nanoparticles with Precise Dimension Control and Tunable Optical Properties, J. Am. Chem. Soc., 2017, 139, 12956-12967. 
11 S. T. Kochuveedu, D.-P. Kim and D. H. Kim, SurfacePlasmon-Induced Visible Light Photocatalytic Activity of TiO2 Nanospheres Decorated by $\mathrm{Au}$ Nanoparticles with Controlled Configuration, J. Phys. Chem. C, 2012, 116, 2500-2506.

12 W. Hou and S. B. Cronin, A Review of Surface Plasmon Resonance-Enhanced Photocatalysis, Adv. Funct. Mater., 2013, 23, 1612-1619.

13 H. Choi, S.-J. Ko, Y. Choi, P. Joo, T. Kim, B. R. Lee, J.-W. Jung, H. J. Choi, M. Cha and J.-R. Jeong, Versatile surface plasmon resonance of carbon-dot-supported silver nanoparticles in polymer optoelectronic devices, Nat. Photonics, 2013, 7, 732-738.

14 Y. Oh, J. W. Lim, J. G. Kim, H. Wang, B.-H. Kang, Y. W. Park, H. Kim, Y. J. Jang, J. Kim, D. H. Kim and B.-K. Ju, Plasmonic Periodic Nanodot Arrays via Laser Interference Lithography for Organic Photovoltaic Cells with $>10 \%$ Efficiency, ACS Nano, 2016, 10, 10143-10151.

15 Y. H. Jang, Y. J. Jang, S. Kim, L. N. Quan, K. Chung and D. H. Kim, Plasmonic Solar Cells: From Rational Design to Mechanism Overview, Chem. Rev., 2016, 116, 14982-15034.

16 S. T. Kochuveedu, Y. H. Jang and D. H. Kim, A study on the mechanism for the interaction of light with noble metalmetal oxide semiconductor nanostructures for various photophysical applications, Chem. Soc. Rev., 2013, 42, 8467-8493.

17 S. Aksu, M. Huang, A. Artar, A. A. Yanik, S. Selvarasah, M. R. Dokmeci and H. Altug, Flexible plasmonics on unconventional and nonplanar substrates, Adv. Mater., 2011, 23, 4422-4430.

18 M. Kahraman, P. Daggumati, O. Kurtulus, E. Seker and S. Wachsmann-Hogiu, Fabrication and characterization of flexible and tunable plasmonic nanostructures, Sci. Rep., 2013, 3, 3396.

19 U. Cataldi, R. Caputo, Y. Kurylyak, G. Klein, M. Chekini, C. Umeton and T. Bürgi, Growing gold nanoparticles on a flexible substrate to enable simple mechanical control of their plasmonic coupling, J. Mater. Chem. C, 2014, 2, 79277933.

20 X. Zhu, L. Shi, X. Liu, J. Zi and Z. Wang, A mechanically tunable plasmonic structure composed of a monolayer array of metal-capped colloidal spheres on an elastomeric substrate, Nano Res., 2010, 3, 807-812.

21 J. Y. Kim, H. Kim, B. H. Kim, T. Chang, J. Lim, H. M. Jin, J. H. Mun, Y. J. Choi, K. Chung, J. Shin, S. Fan and S. O. Kim, Highly tunable refractive index visible-light metasurface from block copolymer self-assembly, Nat. Commun., 2016, 7, 12911.

22 G. Lu, H. Li and H. Zhang, Gold-Nanoparticle-Embedded Polydimethylsiloxane Elastomers for Highly Sensitive Raman Detection, Small, 2012, 8, 1336-1340.

23 J. Singh, H. Chu, J. Abell, R. A. Tripp and Y. Zhao, Flexible and mechanical strain resistant large area SERS active substrates, Nanoscale, 2012, 4, 3410-3414.

24 C. Park, T. Lee, Y. Xia, T. J. Shin, J. Myoung and U. Jeong, Quick, Large-Area Assembly of a Single-Crystal Monolayer of Spherical Particles by Unidirectional Rubbing, Adv. Mater., 2014, 26, 4633-4638.

25 T. Cesca, N. Michieli, B. Kalinic, A. Sanchez-Espinoza, M. Rattin, V. Russo, V. Mattarello, C. Scian, P. Mazzoldi and G. Mattei, Nonlinear absorption tuning by composition control in bimetallic plasmonic nanoprism arrays, Nanoscale, 2015, 7, 12411-12418.

26 A. Yang, A. J. Hryn, M. R. Bourgeois, W.-K. Lee, J. Hu, G. C. Schatz and T. W. Odom, Programmable and reversible plasmon mode engineering, Proc. Natl. Acad. Sci. U. S. A., 2016, 113, 14201-14206.

27 L. Gao, Y. Zhang, H. Zhang, S. Doshay, X. Xie, H. Luo, D. Shah, Y. Shi, S. Xu, H. Fang, J. A. Fan, P. Nordlander, Y. Huang and J. A. Rogers, Optics and Nonlinear Buckling Mechanics in Large-Area, Highly Stretchable Arrays of Plasmonic Nanostructures, ACS Nano, 2015, 9, 59685975.

28 L. J. Sherry, R. Jin, C. A. Mirkin, G. C. Schatz and R. P. Van Duyne, Localized Surface Plasmon Resonance Spectroscopy of Single Silver Triangular Nanoprisms, Nano Lett., 2006, 6, 2060-2065.

29 N. Félidj, J. Grand, G. Laurent, J. Aubard, G. Lévi, A. Hohenau, N. Galler, F. R. Aussenegg and J. R. Krenn, Multipolar surface plasmon peaks on gold nanotriangles, J. Chem. Phys., 2008, 128, 094702.

30 Y.-F. Huang, D.-Y. Wu, H.-P. Zhu, L.-B. Zhao, G.-K. Liu, B. Ren and Z.-Q. Tian, Surface-enhanced Raman spectroscopic study of p-aminothiophenol, Phys. Chem. Chem. Phys., 2012, 14, 8485-8497.

31 P. B. Johnson and R.-W. Christy, Optical constants of the noble metals, Phys. Rev. B: Solid State, 1972, 6, 4370.

32 S. N. Terekhov, S. M. Kachan, A. Y. Panarin and P. Mojzes, Surface-enhanced Raman scattering on silvered porous alumina templates: role of multipolar surface plasmon resonant modes, Phys. Chem. Chem. Phys., 2015, 17, 3178031789.

33 L. Yang, W. Ruan, X. Jiang, B. Zhao, W. Xu and J. R. Lombardi, Contribution of ZnO to Charge-Transfer Induced Surface-Enhanced Raman Scattering in $\mathrm{Au} / \mathrm{ZnO} /$ PATP Assembly, J. Phys. Chem. C, 2009, 113, 117-120.

34 X. Hu, T. Wang, L. Wang and S. Dong, Surface-Enhanced Raman Scattering of 4-Aminothiophenol Self-Assembled Monolayers in Sandwich Structure with Nanoparticle Shape Dependence: Off-Surface Plasmon Resonance Condition, J. Phys. Chem. C, 2007, 111, 6962-6969.

35 P. Xu, L. Kang, N. H. Mack, K. S. Schanze, X. Han and H.-L. Wang, Mechanistic understanding of surface plasmon assisted catalysis on a single particle: cyclic redox of 4-aminothiophenol, Sci. Rep., 2013, 3, 2997.

36 L. V. Brown, H. Sobhani, J. B. Lassiter, P. Nordlander and N. J. Halas, Heterodimers: Plasmonic Properties of Mismatched Nanoparticle Pairs, ACS Nano, 2010, 4, 819-832.

37 P. J. Schuck, D. P. Fromm, A. Sundaramurthy, G. S. Kino and W. E. Moerner, Improving the Mismatch between Light and Nanoscale Objects with Gold Bowtie Nanoantennas, Phys. Rev. Lett., 2005, 94, 017402. 